Annuaire suisse de politique de développement

\title{
Une société de l'information équitable : une question de volonté politique
}

\section{Chantal Peyer}

\section{(2) OpenEdition}

1 Journals

Édition électronique

URL : http://journals.openedition.org/aspd/564

DOI : 10.4000/aspd.564

ISSN : 1663-9669

Éditeur

Institut de hautes études internationales et du développement

\section{Édition imprimée}

Date de publication : 1 novembre 2003

Pagination : 131-133

ISSN : 1660-5934

\section{Référence électronique}

Chantal Peyer, «Une société de l'information équitable : une question de volonté politique », Annuaire suisse de politique de développement [En ligne], 22-2 | 2003, mis en ligne le 22 mars 2010, consulté le 08 septembre 2020. URL : http://journals.openedition.org/aspd/564 ; DOI : https://doi.org/10.4000/ aspd.564 


\title{
Une société de l'information équitable: une question de volonté politique
}

\author{
Chantal Peyer*
}

«

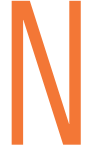
ous ne sommes pas dupes de l'idéologie techniciste, néolibérale et bourrée d'illusions égalitaristes du village planétaire: le fossé technologique et de compétences fait qu'Internet n'est pas la connaissance pour tous, mais bien une démonstration de plus d'un monde à deux vitesses », écrivait en 2000 Olivier Barlet, dans un éditorial d'Africultures ${ }^{1}$. Deux ans plus tard, à l'orée du Sommet mondial sur la société de l'information (SMSI), qu'en est-il? Comment sont perçus les potentiels et les dangers de la société de l'information?

Pour Pain pour le prochain, les nouvelles technologies de l'information et de la communication (NTIC) peuvent être une chance. Le choix de technologies sans fil par exemple - communément appelées WiFi - permet de limiter l'investissement dans des infrastructures coûteuses et rapidement obsolètes $^{2}$. Le développement des moyens de communication facilite la création de réseaux de base et peut renforcer la participation des citoyens. Des applications telles que la télémédecine et l'éducation à distance, ou encore la création de centres d'accès communautaires sont des outils importants de lutte contre la pauvreté.
Mais les NTIC sont des moyens et non une fin; sans volonté politique claire, sans changement de paradigme, l'avènement d'un «village global» égalitaire est une illusion. Aujourd'hui, par exemple, seuls 5\% de la population mondiale - dont $88 \%$ habitent dans les pays du Nord - utilisent Internet. Et au Sud, les usagers de la toile sont en majorité des hommes, issus des zones urbaines et ayant un bon niveau de formation. Dans ses développements actuels, la société de l'information renforce donc les fractures économiques, sociales et culturelles existantes.

La volonté politique sera-t-elle au rendez-vous du Sommet mondial sur la société de l'information? Les résultats des premières conférences préparatoires permettent d'en douter. Pour Pain pour le prochain, les contours de la déclaration adoptée à Genève lors de la première phase du sommet devraient se fonder sur des cadres juridiques existants, notamment la Déclaration universelle des droits de l'homme (1948). En affirmant que «tout individu a droit à la liberté d'opinion et d'expression » et que «ce droit inclut la liberté d'exprimer sans contraintes des opinions et de rechercher, recevoir et produire

* Chargée de programme à Pain pour le prochain et membre de la délégation suisse au Sommet mondial sur la société de l'information, Suisse.

Africultures, Internet en Afrique, $\mathrm{n}^{\circ} 23$, décembre 1999, <www.africultures.com>.

2 Alan Levy, Matching New WiFi Technology with Virtual Private Networks to Create Affordable Universal Internet Access, <www.bytesforall.org/index_connectivity_top1.htm>. 
des informations et des idées à travers tous types de médias et toutes frontières », l'article 19 de cette déclaration résume les revendications essentielles de la société civile: accès pour tous aux moyens de communication (infrastructures) ainsi qu'à la réception et à la production de l'information (contenus). Si les gouvernements sont plus ou moins unanimes à reconnaître ces principes, la mise en œuvre pose cependant problème. Pour trois raisons au moins.

Premièrement, comme l'a rappelé dès sa fondation à fin 2002 la Plate-forme suisse pour la société de l'information - une coalition d'organisations non gouvernementales et de journalistes engagés dans le processus du SMSI -, le respect d'un droit à la communication nécessite de replacer l'homme au centre des préoccupations. Or, dans le processus du SMSI, «l'accent est mis sur les infrastructures (pour le Sud) et les marchés potentiels (pour le Nord) plus que sur les droits et besoins réels des personnes et des communautés $»^{3}$.

Ensuite, la construction d'une société de l'information démocratique et participative nécessite un débat de fond sur des questions comme les droits de propriété intellectuelle, le respect des droits de l'homme et de la liberté d'expression, ou encore la concentration des médias, thèmes qui sont tous au cœur des préoccupations de la société civile 4 . Or ces sujets n'occupent qu'une place mineure, voire sont évincés a priori des négociations officielles du sommet.

Enfin, le manque d'engagements concrets se fait ressentir: pour réduire la fracture numérique et permettre l'accès de tous aux moyens et aux contenus de l'information, des politiques publiques novatrices et des ressources financières conséquentes seront nécessaires. Or, tant la proposition de créer un fonds international de solidarité pour la société de l'information que le soutien apporté aux logiciels libres - pour ne citer que ces deux exemples - peinent à trouver un écho réel dans le cadre du SMSI. Les recommandations faites aux pays en développement pour lutter contre la fracture numérique vont toutes dans le même sens: privatiser les télécommunications, mettre en place des cadres réglementaires stables et attractifs pour les entreprises privées, et s'engager dans des partenariats privés-publics. Pourtant, les politiques de privatisation et de libéralisation actuelles - menées sous l'égide de l'Organisation mondiale du commerce et de l'Union internationale des télécommunications (UIT) - ont montré leurs limites: elles ne permettent pas de développer des infrastructures appropriées, à des coûts abordables, pour les régions et les populations défavorisées des pays en développement ${ }^{5}$.

Pour construire un réel partenariat et trouver des solutions aux problèmes posés par la société de l'information,

3 Site de la Plate-forme suisse pour la société de l'information: <www.comunica-ch.net>.

4 Voir, par exemple, la campagne CRIS (Communication Rights in the Information Society), <www. crisinfo.org $>$.

5 Voir à ce sujet les articles de Jean-Louis Fullsack, notamment L'UIT, la vieille dame des télécommunications dans la tourmente libérale, <www.globenet.org/csdptt>, ou les articles d'Annie ChéneauLoquay sur $<$ www.africanti.org $>$. 
il devient donc urgent d'inventer de nouvelles formes de collaboration et de consultation, voire de gouvernance. Plus que dans tout autre domaine, la société civile et le secteur privé ont un savoir-faire et une créativité qui doivent être pris en compte dans la construction à venir de la société de l'information. C'est pourquoi la Plate-forme suisse a appelé de ses vœux la création d'une taskforce suisse de haut niveau, réunissant tous les acteurs susceptibles d'apporter leur contribution au processus du sommet (responsables des offices compétents, parlementaires, représentants du secteur privé, de la société civile et des médias). Authentiquement tripartite, permanente et transparente, une telle taskforce serait le lieu où notamment se négocient et s'élaborent les positions et propositions suisses pour le sommet. En acceptant cette proposition, le gouvernement suisse pourrait montrer l'exemple et respecter au mot les textes officiels à l'origine du Sommet, qui demandaient l'élaboration de nouvelles formes de consultation tripartite ${ }^{6}$.

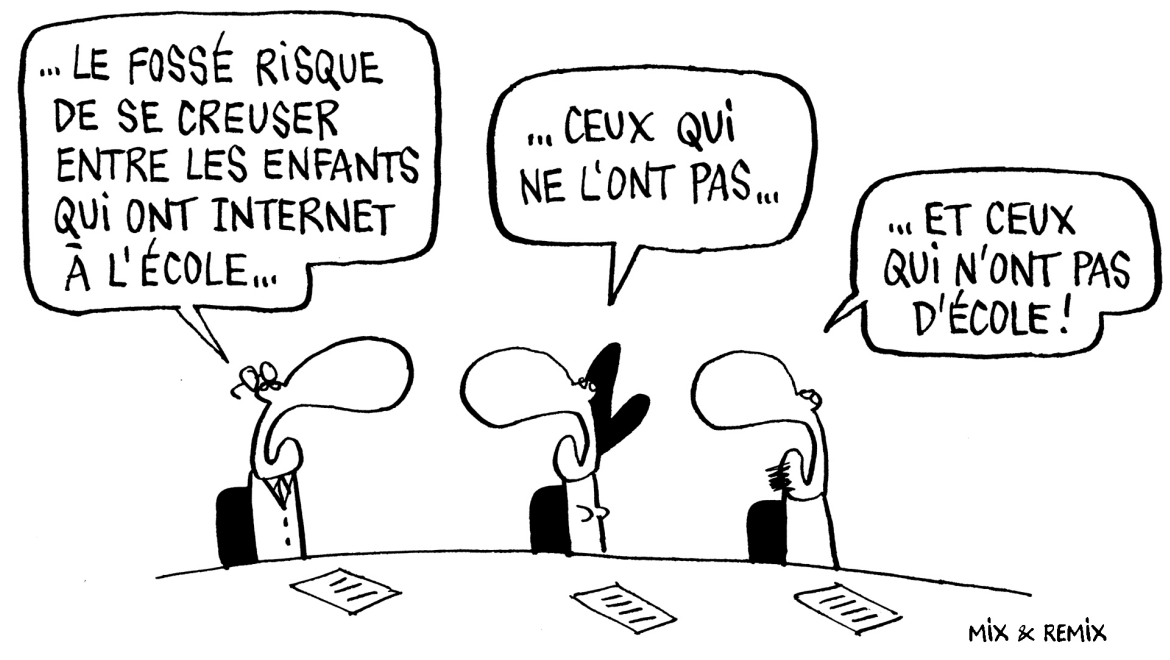

6 Voir, par exemple, la résolution 56/183 de l'Assemblée de l'ONU qui encourage «les organisations non gouvernementales et le secteur privé à contribuer et participer activement au processus intergouvernemental du sommet, ainsi qu'au sommet proprement dit». 